\title{
Overview of techniques applicable to self-interference incoherent digital holography
}

\section{J. Hong \\ jisoohong@mail.usf.edu}

M. K. Kim
Department of Physics, University of South Florida, Tampa, FL 33620, USA

Department of Physics, University of South Florida, Tampa, FL 33620, USA

Self-interference incoherent digital holography (SIDH) retrieves the complex hologram from the object illuminated by the incoherent light. Supported by the adaptive optic feature, SIDH is readily applicable to the ocular imaging to investigate the human retinal cells. Considering the practical issues, issues related to resolution, phase-shifting, and contrast should be addressed to implement the viable SIDH system which is capable of recording the holographic information of human retinal cells under the incoherent illumination. Super resolution image reconstruction technique can be directly applied to SIDH to enhance the resolution of the system without any change of configuration. We present the improved way to incorporate the phase-shifting itself into the lateral shift required by the super resolution technique. To deal with the phase-shifting issue, we present an arbitrary phase shift retrieval algorithm which can reduce the number of phase-shift and accept the blind phase-shift. The single-shot imaging is also possible by adopting the off-axis configuration of SIDH. We will provide the detailed procedures to retrieve the complex hologram using the proposed arbitrary phase shifting algorithm and the off-axis configuration. [DOI: http://dx.doi.org/10.2971/jeos.2013.13077]

Keywords: Digital holography, incoherent holography, retinal imaging, super resolution, single-shot imaging

\section{INTRODUCTION}

Self-interference incoherent digital holography (SIDH) is one of the techniques that can record the holographic information from the object illuminated by the incoherent light. The invention of laser opened the door to the realization of the holography, however the coherent illumination brought many practical problems for the wide application. Hence, the possibility of the incoherent holography was already studied from the early years of the history of holographic imaging [1]-[3]. However, it was not successful until the development of the electronic devices and computer science. Recently, based on the digital holography, many techniques, which can successfully record the holographic information with incoherent illumination, had been reported [4]-[8]. One of the practical approaches is to use the self-interference by splitting the light from the object into the object and the reference beam. Many different configurations including SIDH had been developed to implement this general idea [6]-[8].

The optical configuration of SIDH is a modified Michelson interferometer as shown in Figure 1. SIDH creates the interference based on the self-interfering property. The light from the object is separated to two copies of beams by the beam splitter. After the separation, those two copies of beams meet different mirrors with different curvature (for this illustration, one is plane mirror and the other is the curved mirror). The mixture of them creates the interference at the sensor plane and recorded as an intensity image. Because of the spatial incoherence of the illumination, the interferogram of the extended object will be created by the integration of the interferograms from all the point sources at the object plane. Hence,

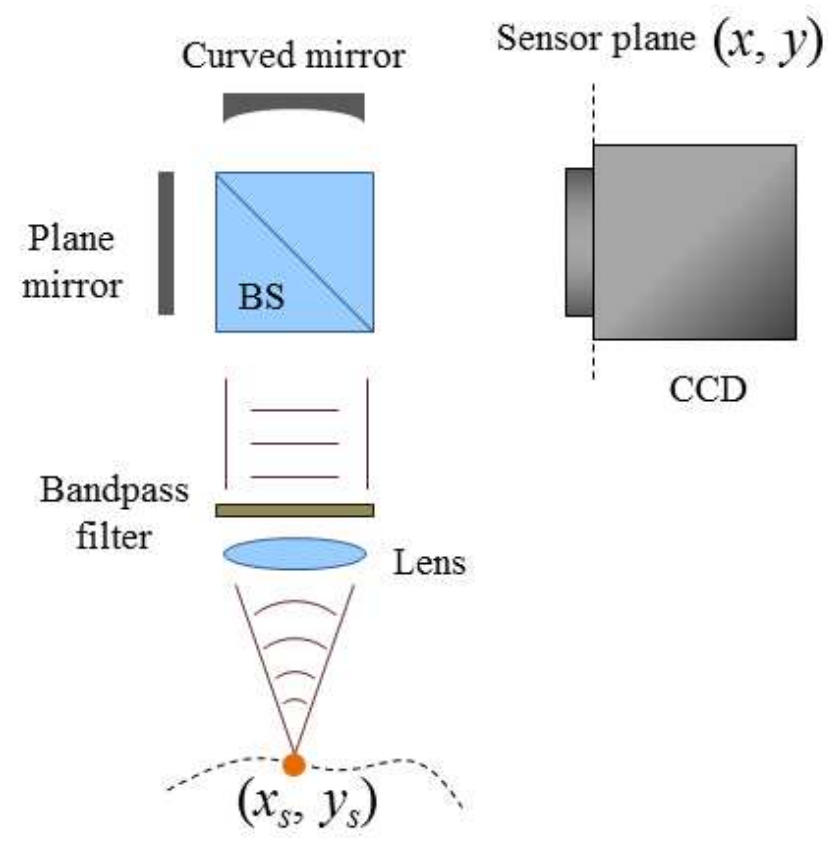

Object

FIC. 1 Optical configuration of SIDH.

the recorded interferogram will be:

$$
I(x, y)=\iint A\left(x_{s}, y_{s}\right)\left\{1+\cos \left[\phi\left(x, y ; x_{s}, y_{s}\right)\right]\right\} \mathrm{d} x_{s} \mathrm{~d} y_{s},
$$

where $(x, y)$ and $\left(x_{s}, y_{s}\right)$ are coordinates of the sensor plane 
and the object plane respectively. $A\left(x_{s}, y_{s}\right)$ is the function dependent on the intensity of each object point, $\phi\left(x, y ; x_{s}, y_{s}\right)$ is a quadratic function. To retrieve the complex hologram, three or four step phase-shifting interferometry is usually adopted by moving one of the mirrors. From the retrieved complex hologram, the reconstruction of the object image can be easily done by applying any propagation method.

The approach using self-interference had shown successful results in many practical applications. For example, Rosen and Brooker had shown the recording of holographic information from the fluorescence object which was not possible with the coherent holography [6]. Recently, our group reported about the full-color holographic camera which can record the holographic information of the natural outdoor scene [9].

In this paper, we will discuss about the possibility of the application of SIDH for the ocular imaging. For the ocular imaging, there are many practical considerations such as resolution, contrast, and phase-shifting. For the resolution enhancement, we propose the way to apply the super resolution image reconstruction algorithm which was developed by the image processing society. And we will also discuss about the arbitrary phase-shifting and off-axis configuration to reduce the number of phase-shifting incorporated in SIDH.

\section{RETINAL IMAGING USING SIDH}

Recently, our group has reported the adaptive optic feature using SIDH which can compensate the aberration which usually appears in the astronomical or ocular imaging [8]. Supported by the adaptive optic feature, the SIDH is readily applicable to the ocular imaging to investigate the retinal structure of the human eye. Because the SIDH does not care about the coherency of the illumination source, we are expecting that the application of SIDH for the retinal imaging can be advantageous than the use of coherent illumination source. As the first step to the retinal imaging by SIDH, we have investigated a half-cut bovine eye sample using the SIDH system illustrated in Figure 1.

We prepared the sample by half-cutting the preserved bovine eye and drying it in the air more than a week. After dried, the sample was cut into many small pieces for easy loading. Figure 2 shows some imaging examples of the prepared bovine eye sample. We can see that the reconstruction at the best focal plane provides the results similar to the direct images. Performing the experiment with the bovine eye, we could list some issues of SIDH system for the retinal imaging.

1. Resolution is always the issue of any types of imaging system. SIDH system is superior to a usual microscope imaging system because it inherently has twice higher resolution as Rosen et al. reported [10]. However, the microscopic application always requires increasing the resolution. Though SIDH itself already has a higher resolution, if there is a way to increase the resolution more, it will be helpful in resolving the structure of retina more clearly.

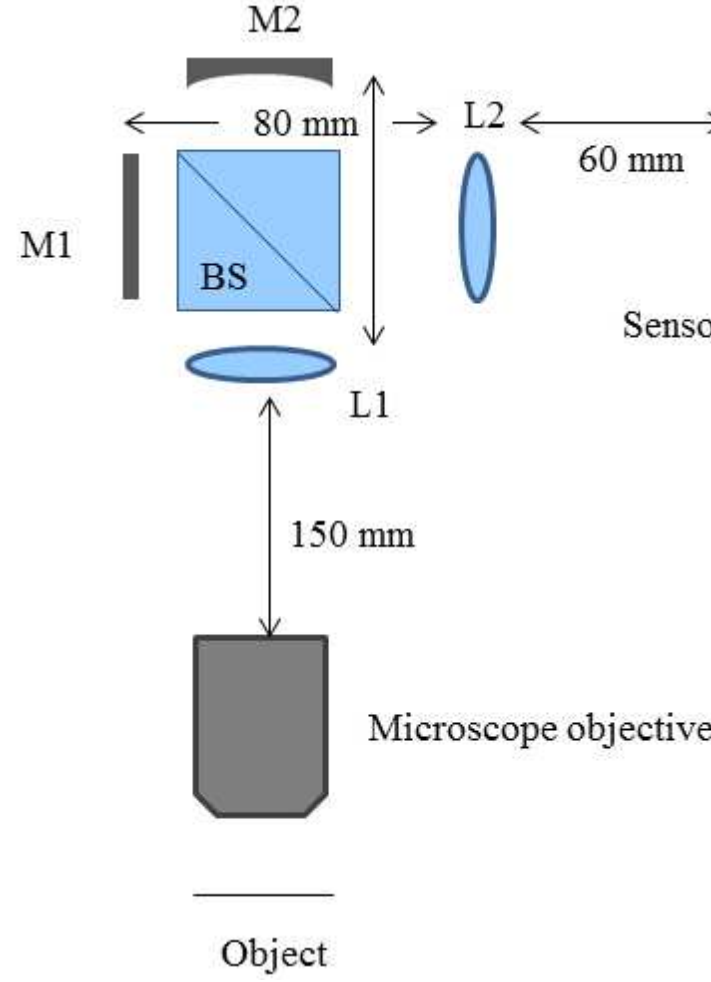

FIG. 2 SIDH system used for retinal imaging. BS: beam splitter, M1: plane mirror, M2: curved mirror with the focal length of $600 \mathrm{~mm}, \mathrm{~L} 1$ and L2: convex lens with the focal length of $50 \mathrm{~mm}$. Microscope objective: 20x, NA $=0.40$.

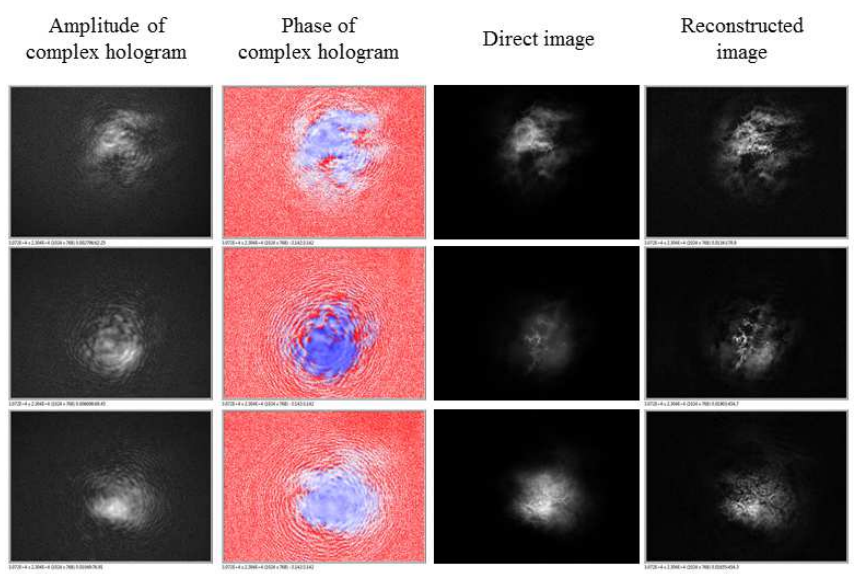

FIG. 3 Experimental data that shows the retinal structures of the half-cut bovine eye. Each row corresponds to different part of the sample.

2. Phase-shifting technique requires multiple exposures for creating one complex hologram, losing temporal resolution in the recorded information. Because the object is required to be nearly stationary during the multiple exposures, the phaseshifting is problematic in the high-speed imaging of the moving object. The human eye is known to be moving all the time even though the subject is doing effort of fixation. Hence the image quality can be affected by the multiple exposures of phase-shifting. For the retinal imaging, reducing the number of phase-shifting will be one of important objectives to improve the image quality.

3. Contrast can be also an issue in applying SIDH for the retinal imaging. The spatial incoherence of the object image makes the DC term of each point source to be added up, re- 


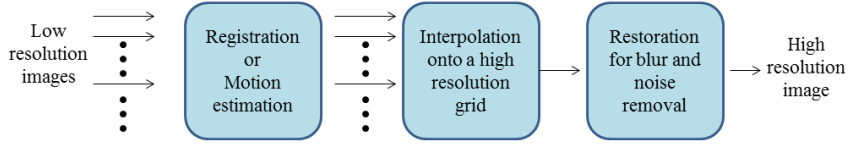

FIG. 4 Process of the SR technique.

sulting in the huge DC in the recorded interferogram. Hence, the dynamic range for the ripples of the recorded interferogram is severely limited, which eventually loses the contrast even though we precisely control the exposure of the recording device.

Among the issues listed above, we are developing techniques to address the first and second issues. In the following sections, we will describe the possible approaches to deal with the resolution and phase-shifting of the SIDH.

\section{SUPER RESOLUTION IMAGE RECONSTRUCTION ALGORITHM}

The image processing society developed a technique, which is called the super resolution (SR) image reconstruction, to record the image with resolution higher than the capability of the recording device (Generally, SR can represent any technique which can overcome the resolution determined by the physical limitation of the recording system. However, in this manuscript, we will use the term SR to refer to the specific scheme which is illustrated in [11]). SR technique records the same scene multiple times with lateral shifts of sub-pixel scale. In this way, the SR technique can record the larger data points of the scene compared with the pixel grids defined by the recording device itself. If we record $n$ laterally shifted images of the scene, the amount of information we obtain is $n$ times larger. Then the high resolution image can be estimated in the $\sqrt{n} \times \sqrt{n}$ times larger pixel grids using a proper algorithm. In practice, though the actual resolution enhancement is not much as the enlarged pixel grid because of the noise and the performance of the adopted algorithm, SR is considered as one of the attractive resolution enhancement techniques because it is applicable to any types of recording devices without change of the basic configuration. Hence, we can think of direct application of SR technique to the conventional SIDH system [12].

In recent decades, a number of algorithms had been developed to implement the concept of SR technique, but the general idea of the entire process can be summarized as the brief process illustrated in Figure 4 [11]. For easy and effective implementation of the sub-pixel movement, the shift of the sensor is supposed to be unknown value. Hence one of the important parts of the algorithm is the estimation of the shift values and the other part is the interpolation of the image in the high resolution grid with the estimated shift values.

The drawback of the SR technique is that it increases the resolution of the recorded image in a time-multiplexing manner. The direct application of SR technique to the SIDH can be done by applying SR algorithm for $n$ laterally shifted recon-
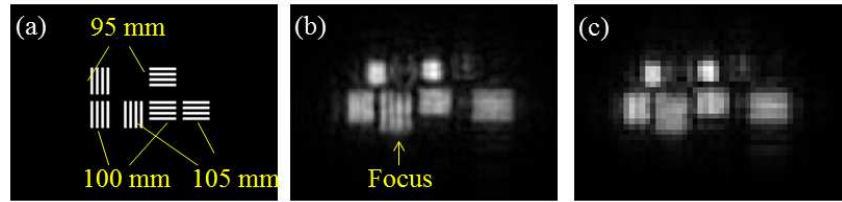

FIG. 5 Simulation results of applying SR technique for the SIDH. (a) The object used for the simulation which is composed of three pairs of gratings located at different distances from the system. The distance of each pair is indicated in the figure. (b) The image reconstructed from the high-resolution hologram whose resolution was enhanced by using the proposed SR technique with 32 exposures. (c) The image reconstructed from the original hologram. For (b) and (c), the reconstruction plane was located at the closet grating (indicated by the arrow in (b)).

structed images. Because SIDH requires phase-shifting to retrieve one complex hologram, if we consider about four-step phase-shifting, totally $4 n$ exposures will be required for the application of SR technique to SIDH. Instead, we can reduce the number of exposures by applying the SR technique at the stage of the phase-shifting. For the four-step phase-shifting, from $0-\pi$ phase-shift pair and $\pi / 2-3 \pi / 2$ phase-shift pair, the DC term of the recorded interferogram can be estimated by $\left(I_{0}+I_{\pi}\right) / 2$ or $\left(I_{\pi / 2}+I_{3 \pi / 2}\right) / 2$ where $I_{\theta}$ represents the interferogram with $\theta$ phase shift. If we let the estimated DC term as $S, I_{\pi}$ can be changed to the form of $I_{0}$ by the relationship:

$$
I_{0}=S-I_{\pi} .
$$

Equivalently, $I_{3 \pi / 2}$ can be changed to $I_{\pi / 2}$ by

$$
I_{\pi / 2}=S-I_{3 \pi / 2} \text {. }
$$

Hence, if we record totally $n$ laterally shifted images for $0-\pi$ phase-shift pair, we can first change all of them to the form of 0 -phase shifted image and then can apply SR algorithm to estimate the image in the $\sqrt{n} \times \sqrt{n}$ times larger pixel grid. The same can be done for the $\pi / 2-3 \pi / 2$ phase-shift pair to estimate the image in the $\sqrt{n} \times \sqrt{n}$ times larger pixel grid. Actually the real part and imaginary part of the complex hologram can be retrieved from $I_{0}$ and $I_{\pi / 2}$ respectively. Hence, by using them, the complex hologram with $\sqrt{n} \times \sqrt{n}$ times larger pixel grid can be estimated.

In this procedure, we record a total of $2 n$ times which is twice effective than the direct application of SR algorithm for reconstructed images. One more advantage of this approach is that it enhances the resolution of the complex hologram instead of the reconstructed image. Because SR algorithm is time consuming process, this approach is easier to change the reconstruction plane.

We performed a preliminary simulation of applying SR technique for SIDH following the schemes described above. As an object, we prepared a set of gratings with the period of $40 \mu \mathrm{m}$ at the reconstruction plane as shown in Figure 5(a). Without SR technique, the pixel pitch of reconstructed image was $80 \mu \mathrm{m}$, and the grating was not resolvable in the reconstructed image (see Figure 5(c)). Using 32 exposures, we could enhance the number of pixels by $4 \times 4$ times larger, reducing the pixel pitch to $20 \mu \mathrm{m}$. However, as explained before, the increase in the number of pixels does not guarantee the resolution enhancement of that amount. Moreover, the sensor movement 
is random and not equally spaced in practice. Hence, the resolution enhancement can differ between horizontal and vertical directions. As a result, in Figure 5(b), we could see that the closest vertical grating is resolvable in the reconstructed image whereas the closest horizontal grating is not.

The next step of the application of SR technique for the SIDH will be taking more advantage from the phase-shifting to reduce the number of exposures. Using the approach introduced in this paper, SR algorithm can be applied only among $0-\pi$ phase-shift pair or $\pi / 2-3 \pi / 2$ phase-shift pair. If it is possible to transform between $0-\pi$ phase-shift pair and $\pi / 2-3 \pi / 2$ phase-shift pair, then the effectiveness will be enhanced by 4 times larger than the application of SR technique for the reconstructed images.

\section{UNKNOWN ARBITRARY PHASE SHIFTING INTERFEROMETRY}

Other than the problem of multiple exposures, the exact phase-shift value is often difficult to achieve in the phaseshifting interferometry because of many practical reasons. Some research groups developed many schemes to take an advantage of phase-shifting in more convenient way [13]-[15]. $\mathrm{Xu}$ et al. reported about the algorithm to retrieve the complex hologram using only two phase-shifted images with arbitrary phase-shift values [15]. In their approach, the number of exposures can be reduced and the exact phase-shift values are not necessary.

Inspired by this approach, the similar procedures can be applied for SIDH to deal with the difficulties coming from the phase-shifting [16]. It also starts from only two phase-shifted images with the arbitrary unknown phase-shift values. If we let two phase shifted images as $I_{1}(x, y)$ and $I_{2}(x, y)$ with phase-shift values $\theta_{1}$ and $\theta_{2}$ respectively, then,

$$
\begin{aligned}
I_{1}+I_{2}= & \iint 2 A\left(x_{s}, y_{s}\right) \\
& \times\left\{1+\cos \left[\phi\left(x, y ; x_{s}, y_{s}\right)+\beta\right] \cos (\alpha)\right\} \mathrm{d} x_{s} \mathrm{~d} y_{s}, \\
I_{1}-I_{2}= & -\iint 2 A\left(x_{s}, y_{s}\right) \\
& \times \sin \left[\phi\left(x, y ; x_{s}, y_{s}\right)+\beta\right] \sin (\alpha) \mathrm{d} x_{s} \mathrm{~d} y_{s},
\end{aligned}
$$

where $\alpha=\left(\theta_{1}-\theta_{2}\right) / 2$ and $\beta=\left(\theta_{1}+\theta_{2}\right) / 2$. Hence, if we know DC term and $\alpha$, the complex hologram will be retrieved by the relationship:

$$
\begin{aligned}
h(x, y)= & \iint 2 A\left(x_{s}, y_{s}\right) \\
& \times \exp \left[i \phi\left(x, y ; x_{s}, y_{s}\right)\right] \exp (i \beta) \mathrm{d} x_{s} \mathrm{~d} y_{s} \\
= & \frac{I_{1}+I_{2}-2 \iint A\left(x_{s}, y_{s}\right) \mathrm{d} x_{s} \mathrm{~d} y_{s}}{\cos (\alpha)}-i \frac{I_{1}-I_{2}}{\sin (\alpha)}
\end{aligned}
$$

Hence, the objective of the algorithm is to estimate the DC term and $\alpha$ from the given phase-shifted images. The algorithm starts from the initial guess of the DC term and $\alpha$. Assuming that the ripple of the interferogram is relatively smaller than DC term, the initial guess of DC term is:

$$
S=2 \iint A\left(x_{s}, y_{s}\right) \mathrm{d} x_{s} \mathrm{~d} y_{s} \approx \frac{\sum_{x, y}\left[I_{1}(x, y)+I_{2}(x, y)\right]}{N M},
$$

where $N$ and $M$ are the number of pixels of the interferogram in $x$ and $y$ directions respectively. For the guess of $\alpha$, we assume that the maximum value of $I_{1}$ and $I_{2}$ will be the same value. If we let the maximum value as $M_{I}$,

$$
\begin{aligned}
& \max \left\{I_{1}+I_{2}-S\right\}=M_{I} \cos (\alpha), \\
& \max \left\{I_{2}-I_{1}\right\}=M_{I} \sin (\alpha) .
\end{aligned}
$$

Hence the initial guess of $\alpha$ is,

$$
\alpha \approx \tan ^{-1}\left[\frac{\max \left(-I_{1}+I_{2}\right)}{\max \left(I_{1}+I_{2}-S\right)}\right] .
$$

Using the initial guesses of the DC term and $\alpha$, the complex hologram can be calculated by Eq. (5). By propagating the complex hologram to the best focal plane, the object image can be reconstructed. From the reconstructed image, two interferogram can be estimated as $\hat{I}_{1}$ and $\hat{I}_{2}$. The estimation of the phase shift values can be done by $l_{2}$-minimization as:

$$
\begin{aligned}
& \hat{\theta}_{1}=\underset{\theta_{1}}{\arg \min } \sum_{x, y}\left|\hat{I}_{1}(x, y)-I_{1}(x, y)\right|^{2}, \\
& \hat{\theta}_{2}=\underset{\theta_{2}}{\arg \min } \sum_{x, y}\left|\hat{I}_{2}(x, y)-I_{2}(x, y)\right|^{2} .
\end{aligned}
$$

Starting from those initial guesses, the algorithm updates the estimated values iteratively. Because of the estimation error, the estimated DC term will be expressed as $\hat{S}=S+\epsilon_{S}$ where $\epsilon_{S}$ is the estimation error. Assuming $\alpha$ is correct,

$$
\hat{h}(x, y)=h(x, y)-\frac{\epsilon_{s}}{\cos (\alpha)} .
$$

Then the reconstruction from this hologram will be:

$$
I_{o}\left(x_{s}, y_{s}\right)-\frac{\epsilon_{s}}{\cos (\alpha)}
$$

where $I_{o}\left(x_{s}, y_{s}\right)$ is the actual object image. By backpropagating this estimated object image to the sensor plane with the phase shift values, the interferograms can be estimated as:

$$
\begin{aligned}
\hat{I}_{1}= & I_{1} \sum_{x_{s}, y_{s}} C\left(-\frac{\epsilon_{s}}{\cos (\alpha)}\right) \\
& \times\left\{1+\cos \left[\phi\left(x, y ; x_{s}, y_{s}\right)+\theta_{1}\right]\right\}, \\
\hat{I}_{2}= & I_{2}+\sum_{x_{s}, y_{s}} C\left(-\frac{\epsilon_{s}}{\cos (\alpha)}\right) \\
& \times\left\{1+\cos \left[\phi\left(x, y ; x_{s}, y_{s}\right)+\theta_{2}\right]\right\},
\end{aligned}
$$

where $C$ is a complex constant which is determined by the system parameters. Hence,

$$
\frac{\sum_{x, y}\left(\hat{I}_{1}+\hat{I}_{2}\right)}{N M} \approx \hat{S}-2 C\left(\frac{\epsilon_{S}}{\cos (\alpha)}\right)+\epsilon_{s}^{\prime} .
$$

Neglecting $\epsilon_{S}^{\prime}$

$$
\epsilon_{s} \approx \frac{\cos (\alpha)}{2 C}\left[\hat{S}-\frac{\sum_{x, y}\left(\hat{I}_{1}+\hat{I}_{2}\right)}{N M}\right] .
$$

Hence, the update for the estimation of $S$ will be done by

$$
\hat{S}_{n+1}=\hat{S}_{n}-\frac{\cos (\alpha)}{2 C}\left[\hat{S}-\frac{\sum_{x, y}\left(\hat{I}_{1}+\hat{I}_{2}\right)}{N M}\right] .
$$



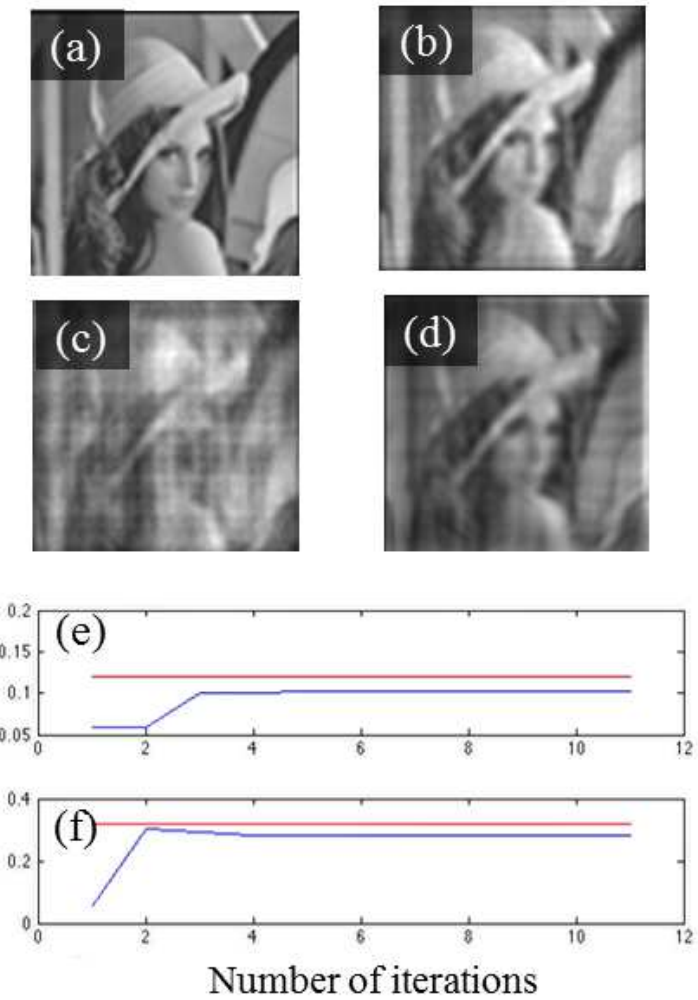

FIG. 6 (a) Object image used for simulation results shown in (b)-(d). (b)-(c): Simulation results of reconstructed image obtained from SIDH with (b) evenly spaced 4-phaseshifted values $(0, \pi / 2, \pi$, and $3 \pi / 2)$ and (c) unknown arbitrary phase-shifted values (here, $0.9796 \pi, 0.8912 \pi, 1.2926 \pi$, and $1.4188 \pi$ ). (d) Simulation result of applying eleven iterations of the proposed algorithm for two interferograms with unknown phase shift values $\left(\theta_{1}=0.12 \pi\right.$ and $\theta_{2}=0.32 \pi$ ). (e) $\hat{\theta}_{1} / \pi$ versus the number of iterations. ( $f$ ) $\hat{\theta}_{2} / \pi$ versus the number of iterations. For (e) and ( $f$ ), the red graphs are the actual phase-shift values and the blue graphs are the estimations.

For the update of the phase shift values, we use Eq. (9) with the updated $S$. By doing the iteration between the update for $S$ and the update for phase shift values, we can approach to the solution of the object image and phase-shift values.

Figure 6(b) shows the simulation result of reconstructed image when 4 phase-shifted values are exactly $0, \pi / 2, \pi$, and $3 \pi / 2$. If the phase-shifted values are unknown arbitrary values, the reconstruction result will be severely distorted as shown in Figure 6(c). Figure 6(d)-(f) show the simulation results of applying the proposed algorithm for two interferograms with the arbitrary phase shift values. After eleven iterations, we can see that the estimation for the object image and phase shift values approach to the solution. We expect that this approach can be helpful in dealing with the practical problems coming from the phase shifting. However, the proposed algorithm is very time consuming because the minimization should be performed at every iteration. And it was assumed that the object to be planar image. Hence, the algorithm needs more improvement to deal with those issues.

\section{OFF-AXIS SIDH CONFIGURATION FOR THE SINGLE-SHOT RECORDING}

Off-axis configuration was adopted in the classical coherent holography to resolve the problem of the on-axis configura-

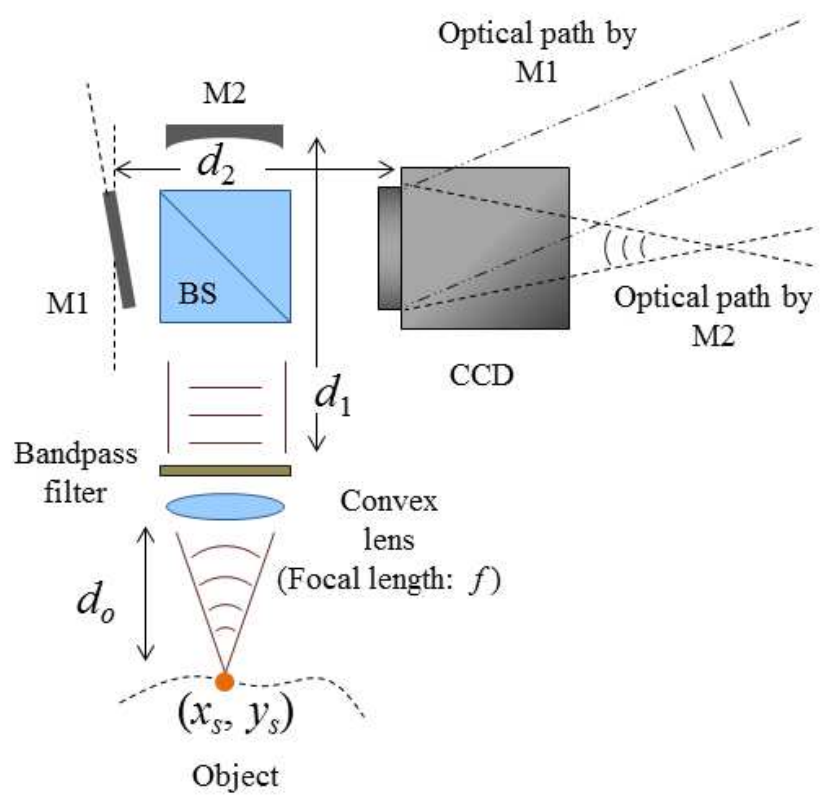

FIG. 7 Optical configuration for off-axis SIDH. BS: beam splitter, M1: plane mirror, M2: curved mirror

tion. In the on-axis configuration, all mixtures of DC term and two conjugate spectral components are recorded as a hologram, resulting in the crosstalk in the reconstruction. By using the off-axis configuration, those components could be separated in the reconstruction showing the clear image. Because the conventional SIDH is also an on-axis configuration, the phase-shifting interferometry was adopted to retrieve the complex hologram free from crosstalk problem. Hence, it is also possible to think about applying the off-axis configuration for the SIDH to obtain the clear reconstructed image without the help of phase-shifting.

Kelner et al. proposed a way to record the single-shot Fourier hologram under the incoherent illumination using two phase-SLMs by configuring $4 f$-like path for one of split beam paths [17]. The drawback of this approach is that it uses two phase-SLMs and the entire optical path becomes relatively larger than the conventional setup.

Recently our group has developed an off-axis SIDH configuration which can retrieve the Fresnel hologram by singleshot imaging. Figure 7 shows the optical configuration of the newly proposed off-axis SIDH. As we can see from Figure 7, the entire setup is nearly the same with the optical configuration of the conventional SIDH except for the slight tilt of the plane mirror. Because of the tilt of the plane mirror, the reflection from the plane mirror will have a certain angle with the reflection from the curved mirror. Hence, if we consider the interference of the two copies of beams originated from a point source object, then the interferogram will be:

$$
I(x, y)=A\left(x_{s}, y_{s}\right)\left|Q\left(x, y ; x_{s}, y_{s}\right)+L\left(x, y ; x_{s}, y_{s}\right)\right|^{2},
$$

where $Q\left(x, y ; x_{s}, y_{s}\right)$ and $L\left(x, y ; x_{s}, y_{s}\right)$ are respectively quadratic and linear phase functions dependent on $(x, y)$ and $\left(x_{s}, y_{s}\right)$.

However, because the illumination source of SIDH is supposed to be incoherent, the strength of interference of two 


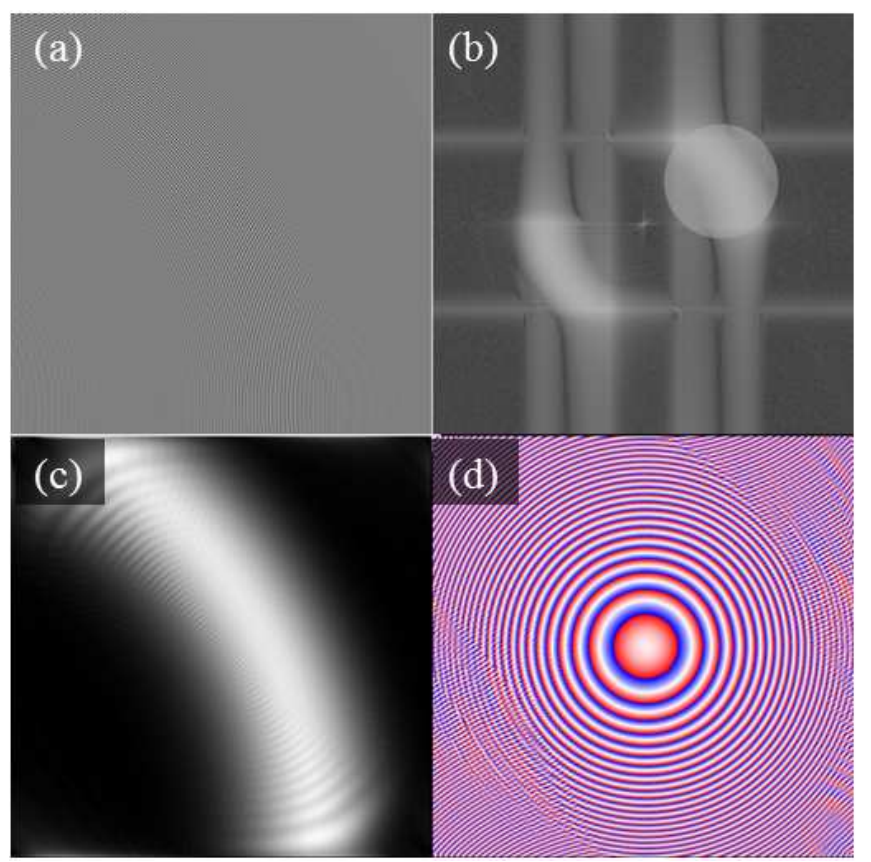

FIG. 8 (a) The synthetic interferogram $(700 \times 700)$ obtained by the point source object and (b) its angular spectrum. The brighter circle is a filter used for extracting one of the spectral components. (c) Amplitude and (d) phase of the retrieved complex hologram.

copies of beams depends on the phase difference between two beams. Hence, the recorded interferogram will be:

$$
I(x, y)=A\left(x_{s}, y_{s}\right)\left(2+P(x, y)\left[Q \cdot L^{*}+Q^{*} \cdot L\right]\right),
$$

where $P(x, y)$ is the envelope function for the interference defined as:

$$
P(x, y)=\exp \left[-\gamma \cdot \Delta(x, y)^{2}\right] .
$$

Here, $\Delta(x, y)$ is the phase difference between two copies of beams and $\gamma$ is a constant related to the temporal coherence length of the illumination. From Eq. (17), we can see that the recorded interferogram separates DC term and two spectral components in the angular spectrum domain.

To retrieve the complex hologram from this interferogram, the recorded intensity image is moved to the angular spectrum domain as shown in Figure 8(b). Using an appropriate filter, we can extract one of the spectral components in the angular spectrum domain. The inverse Fourier transform can transforms the extracted spectral component into the spatial domain, obtaining the complex hologram. As shown in Figure 8(c), the amplitude of the retrieved complex hologram becomes an envelope function of the interference as expected. As shown in Figure 8(d), the phase of the retrieved complex hologram is the quadratic phase function but the information is lost at the area where the envelope function is under the noise. For the extended object, the interferogram is just an integration of the interferograms of all the point sources in the object plane. Hence, the same procedures can be applied for the interferogram of the extended object to retrieve the complex hologram.

After obtaining the complex hologram, the object image can be reconstructed by applying the usual propagation methods such as the angular spectrum and the Fresnel propaga-

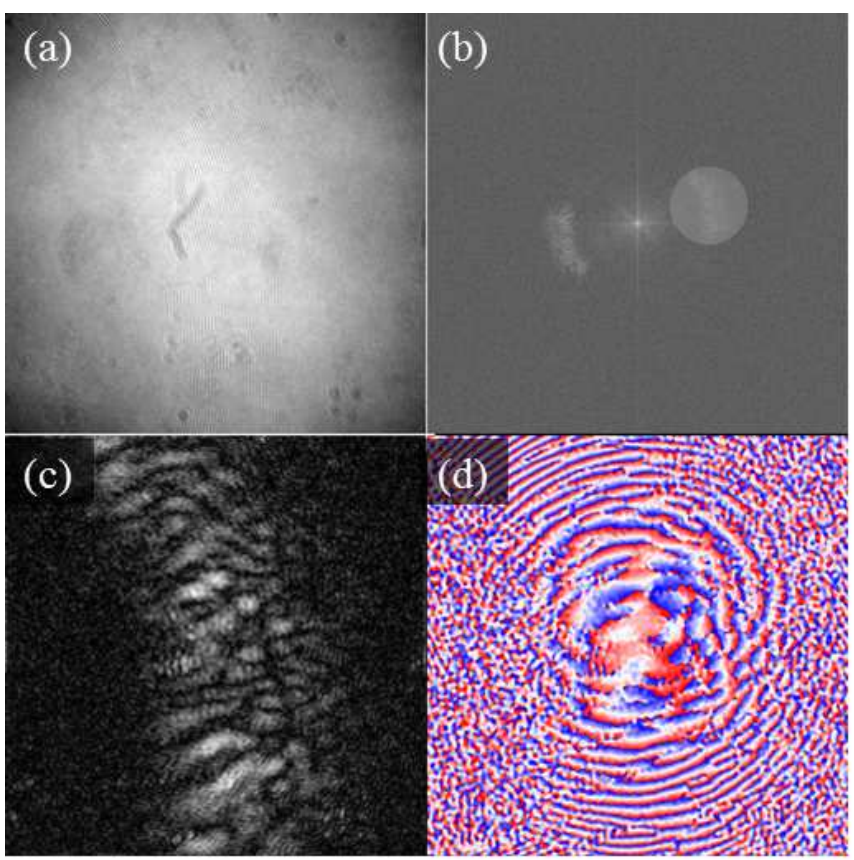

FIG. 9 (a) The interferogram $(700 \times 700)$ obtained from the extended object illuminated by LED (wavelength: $625 \mathrm{~nm}$ ) and (b) its angular spectrum. The brighter circle is a filter used for extracting one of the spectral components. (c) Amplitude and (d) phase of the retrieved complex hologram.
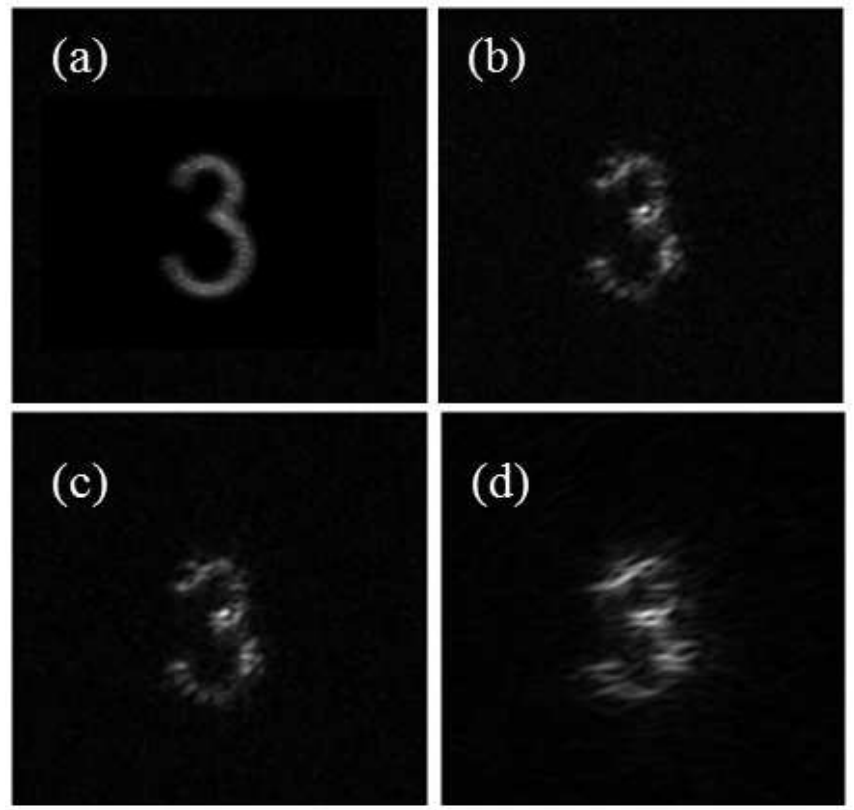

FIG. 9 (a) Baseline image obtained by the normal camera. (b)-(d) Reconstruction results from the complex hologram shown in Figure 9 by using various reconstruction methods: (b) angular spectrum, (c) Fresnel propagation, and (d) cross-correlation with the guide-star hologram (synthetic guide-star hologram was used).

tion. However, the phase of the complex hologram retrieved from the point source object using the off-axis SIDH is an imperfect quadratic phase function. Hence, if we consider this imperfect quadratic phase function as a guide-star hologram of the given system, it will be able to think about the cross-correlation with this guide-star hologram as an optional method for the reconstruction.

Figure 9 shows the experimental example of retrieving 
complex hologram from the interferogram obtained by the extended object. Using the complex hologram shown in Figure 9(c) and 9(d), we reconstructed the object image at the best focal plane using three different reconstruction methods: angular spectrum, Fresnel propagation, and the cross-correlation with the guide-star hologram. Figure 10 compares the reconstruction results by those different reconstruction methods. From this figure, we can see that all of three reconstruction methods show the successful reconstruction using the complex hologram retrieved from the single-shot recording. However, as the number of point sources at the object plane increases, the noise is inevitably increases because of the increased DC term. Hence, considering the discussion of [8] (cross-correlation is better reconstruction method when there is noise or aberration), the cross-correlation with guide-star hologram will be better reconstruction method for the extended object case.

The image quality of reconstruction result shown in Figure 9 is noisier and blurry compared to the result shown in [17]. The reduced image quality may come from the fact that the phase of the guide-star hologram is an imperfect quadratic phase function. If the envelope function is larger than the noise inside the whole field of view, the phase of the guide-star hologram will not lose the information. One of the approach to enlarge the envelope function is to narrow the bandwidth of the bandpass filter in the optical setup to increase the temporal coherence. However, it will lower down the intensity of the input light to the sensor, making SNR of the interferogram worse. The other possible approach is to adopt the phase SLM instead of the plane mirror implementing the tilt by changing the phase value of the SLM. Because the tilt can be implemented without the mechanical tilt, the phase difference between two copies of beams will be ideally zero. Our future research direction is to enhance the image quality adopting one of those approaches.

\section{SUMMARY AND CONCLUSION}

In this paper, we have investigated the possibility of applying SIDH for the ocular imaging. And we discussed the confronting issues for the practical implementation of retinal imaging system: resolution, phase-shifting, and contrast. We have shown that various approaches can be applied to deal with those issues.

Using the SR technique, the resolution of the reconstructed image can be enhanced without the change of the optical configuration of SIDH. And the resolution can be arbitrarily enhanced by sacrificing the temporal resolution of the information. We have shown that, by taking the exposures used for phase-shifting into the lateral shift required by SR technique, the effectiveness can be increased by twice.

Unknown arbitrary phase retrieval algorithm can be helpful in dealing with the phase-shifting problem of SIDH. By using this approach, the number of phase-shift can be reduced to two and the blind phase-shift is also acceptable. However, the algorithm is time-consuming because of iterations and the ob- ject image is assumed to be planar. For the practical use, more development is required for this approach.

Using off-axis configuration, we have demonstrated a singleshot recording of holographic information under the incoherent illumination. Because of the off-axis configuration, DC term and two spectral components can be separated in the angular spectrum of the recorded interferogram. Hence, the complex hologram can be easily retrieved by extracting one of the spectral components. Because the guide-star hologram of the system is an imperfect quadratic phase function, we also proposed a cross-correlation with the guide-star hologram as one optional reconstruction method.

We expect that the techniques discussed in this paper will resolve many of the practical issues against the widespread use of SIDH.

\section{ACKNOWLEDGEMENT}

Research reported in this publication was supported by the National Eye Institute of the National Institutes of Health under Award Number R21EY021876. The content is solely the responsibility of the authors and does not necessarily represent the official views of the National Institutes of Health.

\section{References}

[1] A. W. Lohmann, "Wavefront reconstruction for incoherent objects," J. Opt. Soc. Am. 55, 1555-1556 (1965).

[2] G. Cochran, "New method of making fresnel transforms with incoherent light," J. Opt. Soc. Am. 56, 1513-1517 (1966).

[3] F. Dubois, L. Joannes, and J. Legros, "Improved three-dimensional imaging with a digital holography microscope with a source of partial spatial coherence," Appl. Optics 38, 7085-7094 (1999).

[4] T.-C. Poon, "Three-dimensional image processing and optical scanning holography," Adv. Imag. Elect. Phys. 126, 329-350 (2003).

[5] Y. Li, D. Abookasis, and J. Rosen, “Computer-generated holograms of three-dimensional realistic objects recorded without wave interference," Appl. Optics 40, 2864-2870 (2001).

[6] J. Rosen, and C. Brooker, "Non-scanning motionless fluorescence three-dimensional holographic microscopy," Nat. Photonics 2, 190-195 (2008).

[7] G. Pedrini, H. Li, A. Faridian, and W. Osten, "Digital holography of self-luminous objects by using a Mach-Zehnder setup," Opt. Lett. 37, 713-715 (2012).

[8] M. K. Kim, "Incoherent digital holographic adaptive optics," Appl. Optics 52, A117-A130 (2013).

[9] M. K. Kim, "Full color natural light holographic camera," Opt. Express 21, 9636-9642 (2013).

[10] J. Rosen, N. Siegel, and G. Brooker, "Theoretical and experimental demonstration of resolution beyond the Rayleigh limit by FINCH fluorescence microscopic imaging," Opt. Express 19, 26249-26268 (2011).

[11] S. C. Park, M. K. Park, and M. G. Kang, "Super-resolution image reconstruction: a technical overview," IEEE Signal Proc. Mag. 20, 21-36 (2003). 
[12] J. Hong, and M. K. Kim, "Resolution enhancement of incoherent digital holography using the super resolution image reconstruction technique," in Digital Holography and Three-Dimensional Imaging, OSA Technical Digest (online), DTh1A.3 (Optical Society of America, Washington, 2013).

[13] C. Guo, L. Zhang, H. Wang, J. Liao, and Y. Zhu, "Phase-shifting error and its elimination in phase-shifting digital holography," 0pt. Lett. 27, 1687-1689 (2002).

[14] S. Zhang, "A non-iterative method for phase-shift estimation and wave-front reconstruction in phase-shifting digital holography," Opt. Commun. 268, 231-234 (2006).

[15] X. F. Xu, L. Z. Cai, Y. R. Wang, X. L. Yang, X. F. Meng, G. Y. Dong, X. $X$. Shen, and $H$. Zhang, "Generalized phase-shifting interferometry with arbitrary unknown phase shifts: direct wavefront reconstruction by blind phase shift extraction and its experimental verification," Appl. Phys. Lett. 90, 121124 (2007).
[16] J. Hong, and M. K. Kim, “Unknown Arbitrary Phase Shift Retrieval and Holographic Reconstruction from Images Obtained from SelfInterference Incoherent Digital Holography," in Frontiers in Optics 2013 , I. Kang, D. Reitze, N. Alic, and D. Hagan, eds., OSA Technical Digest (online), FTh3D.6 (Optical Society of America, Washington, 2013).

[17] R. Kelner, J. Rosen, and G. Brooker, "Enhanced resolution in Fourier incoherent single channel holography (FISCH) with reduced optical path difference," Opt. Express 21, 20131-20144 (2013). 